\title{
Hall Effect in InAs/GaAs Superlattices with Quantum Dots: Identifying the Presence of Deep Level Defects
}

\author{
R.M. Rubinger*, G.M. Ribeiro ${ }^{\dagger}$, A.G. de Oliveira $^{\dagger}$, H.A. Albuquerque ${ }^{\dagger}$, \\ R.L. da Silva ${ }^{\dagger}$, W.N. Rodrigues ${ }^{\dagger}$, and M.V.B. Moreira ${ }^{\dagger}$ \\ * Departamento de Física e Química, Instituto de Ciências, Universidade Federal de Itajubá, \\ Caixa Postal 50, 37500-903, Itajubá, Brazil \\ † Departamento de Física, Instituto de Ciências Exatas, Universidade Federal de Minas Gerais, \\ Caixa Postal 702, 30123-970, Belo Horizonte, MG, Brazil
}

Received on 27 March, 2003

\begin{abstract}
We have carried out van der Pauw resistivity and Hall effect measurements on a series of Molecular Beam Epitaxy InAs/GaAs superlattice samples containing InAs quantum dots. Three growth parameters were varied, the InAs coverage, the number of repetitions of the InAs/GaAs layers, and the GaAs spacer thickness. The results can be grouped in two sets, those samples presenting low and high resistivity. The group presenting low resistivity is composed by the samples with GaAs spacer of 30 monolayers (ML) and InAs coverage of 1.9 monolayers. The group presenting high resistivity is composed of samples with GaAs spacer of $40 \mathrm{ML}$. We claim that the high resistivity characteristic is due to the presence of deep level. Increasing the spacer from 30 to $40 \mathrm{ML}$ decouples the InAs planes favouring the deep level formation.
\end{abstract}

\section{Introduction}

Quantum dots (QD) are quasi-zero-dimensional structures and should behave in some aspects like conventional point defects. InAs QD forms on GaAs due to the lattice mismatch between both materials. Besides the electronic states of the QD itself, deep states related to the InAs layers and QD have also been reported by some researchers [1].

In this work we performed Hall measurements on InAs/GaAs QD in order to investigate the formation of such deep levels, which are standard characteristic of high resistive samples due to their high activation energies.

\section{Experimental details}

We have grown samples stacking InAs/GaAs by Molecular Beam Epitaxy (MBE). The samples were grown on semiinsulating Liquid Encapsulated Czochralsky (LEC) GaAs substrates. A $1 \mu \mathrm{m} \mathrm{GaAs}$ buffer layer was grown at a substrate temperature of $600^{\circ} \mathrm{C}$. After that the growth temperature was reduced and kept between $495^{\circ} \mathrm{C}$ and $530^{\circ} \mathrm{C}$ during the InAs/GaAs growth. Three parameters were intentionally varied, namely, the InAs coverage, GaAs spacer thickness, and the number of periods of InAs/GaAs. Two GaAs spacer thickness of 30 and 40 monolayers (ML) were used. The InAs coverage varied between 1.4ML and 3.0ML. The growth parameters are summarized in Table 1.

Table 1 . The growth parameters.

\begin{tabular}{|c|c|c|c|c|}
\hline Sample & Growth Temp. $\left({ }^{\circ} \mathrm{C}\right)$ & \# InAs & InAs coverage (ML) & GaAs Thick (ML) \\
\hline 9821 & 500 & 1 & 1.9 & 30 \\
\hline 9822 & 530 & 5 & 1.9 & 30 \\
\hline 9823 & 510 & 10 & 1.9 & 30 \\
\hline 9826 & 510 & 50 & 1.9 & 30 \\
\hline 9827 & 510 & 50 & 1.9 & 40 \\
\hline 9829 & 510 & 50 & 2.0 & 40 \\
\hline 9832 & 495 & 50 & 2.5 & 40 \\
\hline 9833 & 510 & 50 & 3.0 & 40 \\
\hline 9902 & 510 & 50 & 1.4 & 40 \\
\hline
\end{tabular}


The contacts were deposited on the surface of the sample in a van der Pauw geometry. For this we used small In drops and backed the contacts in a $\mathrm{N}_{2}: \mathrm{H}_{2}$ 85:15 reducing atmosphere at $300^{\circ} \mathrm{C}$ for 10 minutes. The van der Pauw measurements followed the same procedures of Look [2]. The measurements were carried in darkness. For low resistive samples it was possible to measure from room temperature down to $3.9 \mathrm{~K}$, while for high resistive samples the lowest temperature was around $250 \mathrm{~K}$.

\section{Results}

We obtained the Hall density $n$ and the resistivity $\rho$ as a function of the temperature. For obtaining these parameters the sample was considered as a two-dimensional electron gas (2DEG). The samples can be grouped in two sets according to their electrical characteristics, either high or low resistivity values. We will assign them as group \#1 and group \#2 respectively. Group \#1 is composed by samples $98-21,22,23$, and 26. Group \#2 is composed by samples 98-27, 29, 32, 33 and 9902.

Figure 1 shows the planar density $n$ as a function of temperature for the group \#1. It presents the same behavior for all samples. It decreases slightly with the temperature, having a minimum in the range between $15 \mathrm{~K}$ and $60 \mathrm{~K}$ and increases for further decreasing of the temperature. This is a typical 2DEG signature.

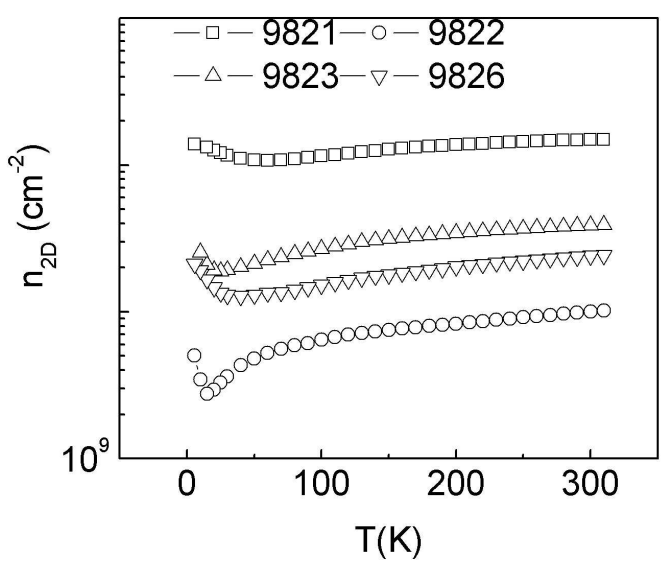

Figure 1. The $n$ function for group \#1.

Figure 2 presents the resistivity results for group \#1, measured in units of $\Omega$ [], where [] stands for adimensional unit since the electron gas is confined in two dimensions. The resistivity values are in the range of $4 \times 10^{4} \Omega[]$ to $2 \times 10^{7} \Omega[]$.

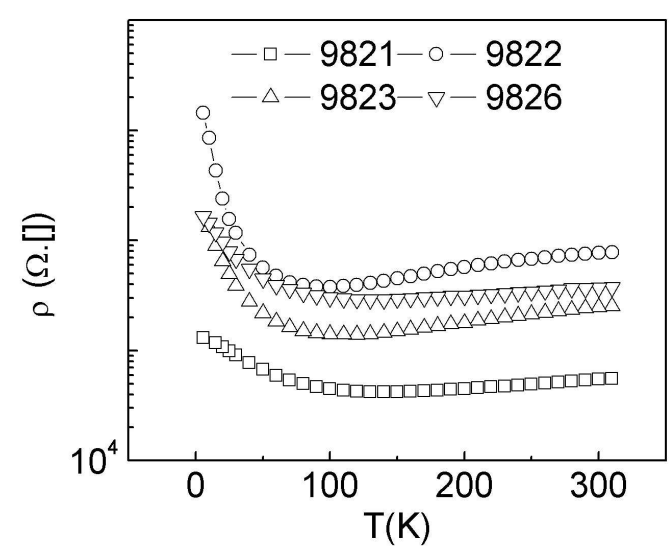

Figure 2. The resistivity function for group \#1.

The resistivities decrease for increasing temperatures reaching a minimum around $100 \mathrm{~K}$ and then increase slightly for further temperature elevation.

Figure 3 presents the planar $n$ for the group \#2. Although there is no $2 \mathrm{D}$ signature for this group this was carried to compare with results of group \#1. All samples present the same behavior, increasing $n$ for increasing temperature. The 2D free carrier density is of $n$ type and temperature dependent measurements give activation energies between $0.4 \mathrm{eV}$ and $0.6 \mathrm{eV}$. These values are too high to be related to an electronic state of InAs QDs, which are in the order of $0.2 \mathrm{eV}[3,4]$.

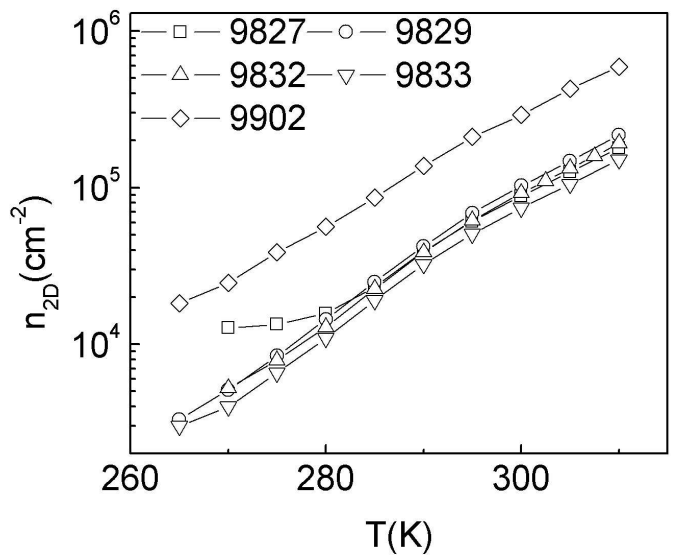

Figure 3. The $n$ function for group \#2.

Figure 4 presents the resistivity results for group \#2. The resistivity values are in the range of $5 \times 10^{10} \Omega[]$ to $5 \times 10^{12} \Omega[]$. All samples of group \#2 present the same behavior with the resistivity decreasing for increasing temperatures. 


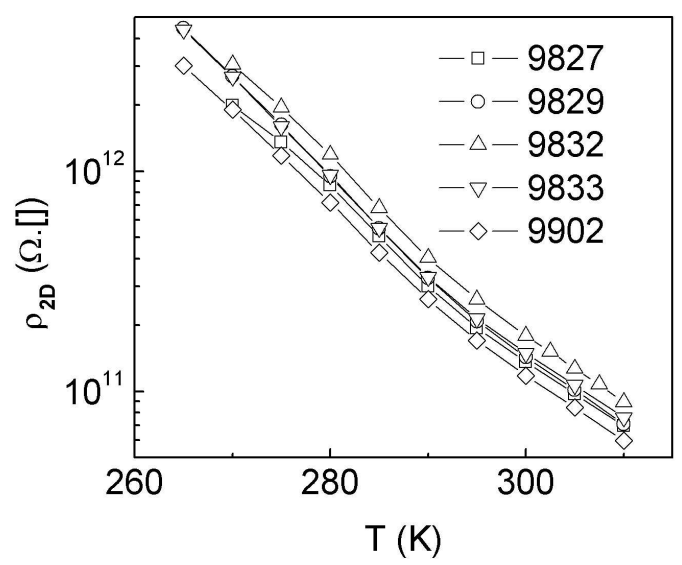

Figure 4. The resistivity function for group \#2.

\section{Discussion}

The experimental results confirm the differences between group \#1 and \#2. It is also important to note that group \#1 is composed of samples with $30 \mathrm{ML}$ GaAs spacers, and group \#2 is built with the $40 \mathrm{ML} \mathrm{GaAs} \mathrm{spacer} \mathrm{samples.}$

Since the samples are quite similar concerning the growth parameters, we claim that deep levels in group \#2 explain the differences. Indeed, the temperature dependence and the electric characteristics of group \#2 which scales with activation energies between $0.54 \mathrm{eV}$ and $0.60 \mathrm{eV}$ can be explained based on the presence of deep levels.

There are three main factors, which would explain the formation of deep levels in our samples. The first factor is related to the $7 \%$ lattice mismatch between InAs and GaAs, which induces the formation of self-assembled quantum dots (SAQD) when the InAs coverage is higher than the critical value of $1.65 \mathrm{ML}$. The strained GaAs layer might result in the presence of deep levels, as have been previously reported [4-6]. The second factor is the growth temperature. Low growth temperatures contribute to the formation of deep level defects [7]. All insulating samples were grown at $510^{\circ} \mathrm{C}$ (except the sample $\# 9832$ that was grown at $495^{\circ}$ ) which is well above $400^{\circ} \mathrm{C}$ and, in turn, results in negligible deep level formation in pure GaAs samples. For such growth temperatures the strain reaches deep into the GaAs layer. This contribute to the formation of deep levels. The third factor is the stacking of layers. It is well known that stacking another InAs layer closer to a first one induces the formation of vertically coupled quantum dots [8]. However, recent works [4-5] indicate another effect of stacking layers, the reduction of the formation of deep levels by lattice relaxation.

All samples of group \#2 have a nominal GaAs layer thickness of $40 \mathrm{ML}$, a number of superlattice repetitions of 50 , a growth temperature of $495^{\circ} \mathrm{C}$ or $510^{\circ} \mathrm{C}$, and InAs coverage between 1.40ML and 3.00 ML. We claim that although the GaAs layer is thin enough to allow the formation of vertically coupled quantum dots it is beyond a critical thickness responsible for the deep level inhibition.

Group \#1 has four samples with the GaAs layer thickness of 30ML. For samples 9821, 9822, 9823 and 9826 the effect of stacking seems to be efficient to inhibit deep defect formation.

\section{Summary}

In this work we have measured Hall effect and resistivity in self-assembled quantum dots samples. Some of them show deep level defect characteristics. We identified that stacking layers of InAs spaced by GaAs layers of 30ML thick inhibits the deep level defect formation.

A GaAs spacer layer thickness of 40ML is enough to preserve the vertically coupled quantum dots [8]. It is known that the proximity of InAs layers plays an important role in deep level inhibition [1]. In our case, although 40ML is not thick enough to decouple the vertically alignment of QD it proved not to be efficient in inhibiting the deep level formation. Under the used growth conditions, there might be a critical value for the GaAs layer thickness between 30 and $40 \mathrm{ML}$ above which the inhibition of deep levels becomes ineffective.

\section{Acknowledgements}

We would like to acknowledge the Brazilian agencies CNPq, CAPES and FAPEMIG for financial support.

\section{References}

[1] C.J. Park, H.B. Kim, Y.H. Lee, D.Y. Kim, T.W. Kang, C.Y. Hong, H.Y. Cho, and M.D. Kim, J. Crys. Growth 227-228, 1057 (2001).

[2] D.C. Look in: Electrical Characterization of GaAs Materials and Devices, Wiley, New York, 1989.

[3] C. Walther, J. Bollmann, H. Kirsme, W. Neumann, and T. Masselink, Appl. Phys. Lett. 76, 2916 (2000).

[4] C. Walther, J. Bollmann, H. Kirsme, W. Neumann, and T. Masselink, Phys. B 273-4, 971 (1999).

[5] L. Dózsa, Zs.J. Horváth, Vo Van Tuyen, B. Pödor, T. Mohácsy, S. Franchi, P. Frigeri, E. Gombia, and R. Mosca, Microelectron. Engineer. 51-52, 85 (2000).

[6] R.M. Rubinger, J.C. Bezerra, E.F. Chagas, J.C. Gonzalez, W.N. Rodrigues, G.M. Ribeiro, M.V.B. Moreira, and A.G. de Oliveira, J. Appl. Phys. 84, 3764 (1998).

[7] N.N. Ledentsov, M. Grundmann, N. Kirstaedter, O. Schmidt, R. Heitz, J. Bohrer, D. Bimberg, V.M. Ustinov, V.A. Shchukin, A.Y. Egorov, A.E. Zhukov, S. Zaitsev, P.S. KopEv, Z.I. Alferov, S.S. Ruvimov, A.O. Kosogov, P. Werner, U. Gosele, and J. Heydenreich, Sol.-Stat. Electron. 40, 785 (1996).

[8] J.C. González, F. M. Matinaga, W.N. Rodrigues, M.V.B. Moreira, A.G. de Oliveira, M. I. N. da Silva,.J. M. Vilela, M. S. Andrade, D. Ugarte, and P. C. Silva; Appl.Phys.Lett., 76 (23), 3400 (2000). 